\title{
CAPRA: piloting biological chain elongation of syngas-based bio-ethanol
}

\author{
Fabian De Wilde*a, Sylvia Gildemyn ${ }^{a}$ and Jan Smis ${ }^{a}$ \\ * presenter, fabian.dewilde@ows.be \\ a OWS nv, Dok-Noord 5, 9000 Gent, Belgium
}

\section{HIGHLIGHTS:}

- Syngas-based bio-ethanol production is coupled to biological caproic acid production to obtain high added-value products from $\mathrm{CO}_{2}$.

- After studying operational parameters at $5 \mathrm{~L}-\mathrm{scale}$, the process was upscaled to a $125 \mathrm{~L}$ bioreactor

- Continuous in-line extraction of the caproic acid allows recovery of caproic acid bio-oil for demonstration of down-stream applications

BACKGROUND: Carbon Capture and Utilisation (CCU) technologies now deliver a wide range of products with varying amounts of incorporated $\mathrm{CO}_{2}$. The downstream processing of these products after an initial production step can be challenging. Initial CCU products are furthermore typically limited to $\mathrm{C} 1$ and $\mathrm{C} 2$ hydrocarbons, for example ethanol in case of a biological reduction process. To tackle both these issues simultaneously, a biological chain elongation technology can transform the short chain products of an initial syngas fermentation step into caproic acid bio-oil, a longer chain product that readily phase-separates. This avoids an energyintensive distillation step for product recovery. The coupling of syngas fermentation and biological chain elongation has been demonstrated at labscale $^{1}$. To bring forward this biological chain elongation technology, several technical challenges remain, including the definition of optimal operational conditions and scale-up challenges. The CAPRA project brings together industry partners and research institutes - ArcelorMittal, OWS, Proviron, Ghent University and VITO - to develop a biological chain elongation process at pilot scale, including: 1) in-line product recovery; 2) development of downstream processing routes to fine chemicals; and 3) an extensive life-cycle assessment and techno-economic assessment of the process.

The specific objectives of the work performed at OWS were: 1) the definition of the optimal substrate ratio (ethanol/acetic acid ratio) for the mixed culture bioprocess; and 2) the scale-up of the bioprocess for the production of minimum $50 \mathrm{~kg}$ caproic acid bio-oil, at a production rate of $10 \mathrm{~g} / \mathrm{L} / \mathrm{d}$.

RESULTS \& DISCUSSION: A lab-scale bioreactor, inoculated with an enriched mixed microbial culture, was operated continuously using substrate with a varying molar ratio of ethanol/acetic acid: $3,6,10,20$. At steady state conditions, and for three independent time periods, the molar ratio of 3 resulted in the highest carbon conversion efficiencies, here calculated as the sum of butyric, caproic and caprylic acid measured in the 
reactor broth (Figure 1 ). This increase in conversion efficiency was mainly a consequence of increased butyric acid production at lower ratios. These findings confirm previous reports, stating that lower molar ratios favour higher conversion rates, while higher molar ratios favour longer chain products $^{1,2}$. This observation is related to the increased ATP production at higher ethanol/acetic acid ratio's, which increases the feasibility of the chain elongation reaction, while the lower ethanol/acetic ratio probably decreases biomass production, favouring the product output ${ }^{1,3}$.

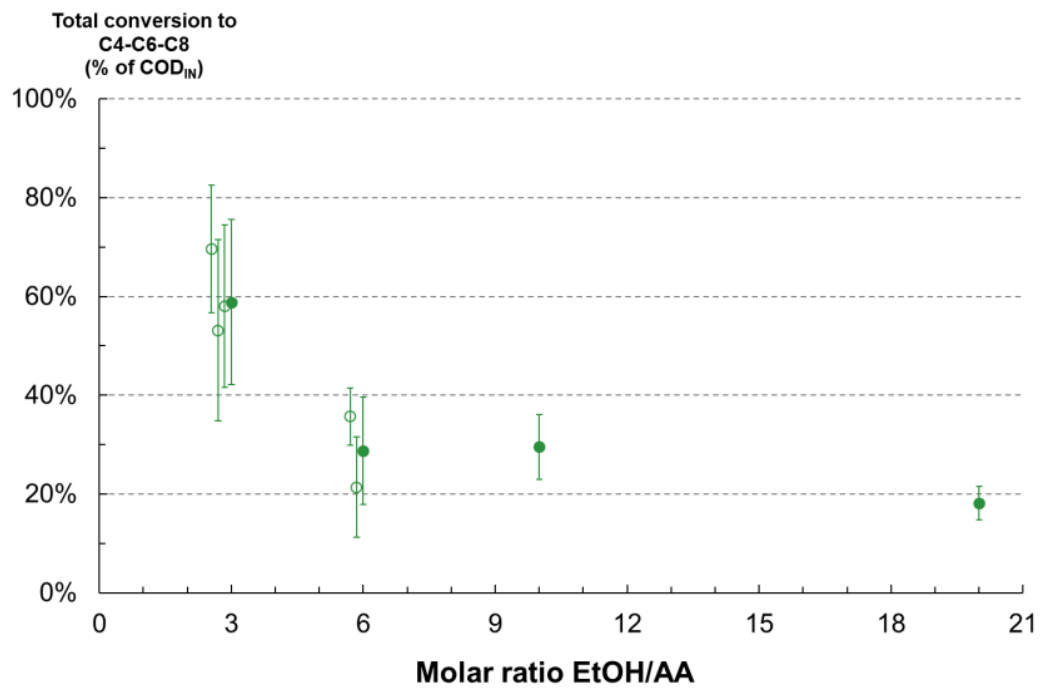

Figure 1. The total conversion efficiency of ethanol and acetic acid to butyric, caproic and caprylic acid was highest at a molar ratio of 3 , with a decreasing trend at higher ethanol/acetic acid ratios.

Based on the conclusions from lab-scale research performed by OWS and Ghent University, a pilot-scale reactor was designed to scale up the bioproduction process. This pilot includes continuous product recovery via in-line membrane-based pertraction. The continuous removal of caproic acid can positively affect the process, by avoiding product inhibition and by increasing the driving force for the production of the more hydrophobic longer chain products.

The pilot scale bioreactor consists of a $125 \mathrm{~L}$ biological compartment, a biomass filtration system to obtain cell-free permeate, and a pertraction system in which the permeate contacts the extraction solvent, following the principles of a system described previously for a lab-scale reactor ${ }^{1}$. The bioreactor was operated continuously using the optimal operational parameters defined earlier in the project. The initial three months of operation resulted in the production of almost $20 \mathrm{~kg}$ caproic acid. A loading rate increase will result in a production rate of $10 \mathrm{~g} / \mathrm{L} / \mathrm{d}$ and the production of at least 50 to $100 \mathrm{~kg}$ extracted caproic acid bio-oil by December 2020 . This production phase deepens the general understanding of biological chain elongation under realistic operational conditions. The extracted caproic acid bio-oil will allow the demonstration of downstream processes, will greatly improve the marketability of the chain elongation process, and will create a non-palm-based caproic acid market. 
CONCLUSION: This study represents a major step forward in bringing biological chain elongation closer to the market. The combination of labscale research on operational parameters and pilot activities under realistic conditions are a unique asset. CAPRA contributes to the advancement of CCU technologies and to solving challenges related to chain elongation technology.

\section{REFERENCES}

1. Gildemyn, S., Molitor, B., Usack, J. G., Nguyen, M., Rabaey, K., \& Angenent, L. T. (2017). Upgrading syngas fermentation effluent using Clostridium kluyveri in a continuous fermentation. Biotechnology for biofuels, 10(1), 83.

2. Hegner, R., Koch, C., Riechert, V., \& Harnisch, F. (2017). Microbiome-based carboxylic acids production: from serum bottles to bioreactors. RSC advances, 7(25), 15362-15371.

3. Angenent, L. T., Richter, H., Buckel, W., Spirito, C. M., Steinbusch, K. J., Plugge, C. M., ... \& Hamelers, H. V. (2016). Chain elongation with reactor microbiomes: open-culture biotechnology to produce biochemicals. Environmental science \& technology, 50(6), 27962810. 